\title{
Intuitive understanding of non-gaussianity in ekpyrotic and cyclic models
}

\author{
Jean-Luc Lehners ${ }^{1}$ and Paul J. Steinhardt ${ }^{1,2}$ \\ ${ }^{1}$ Princeton Center for Theoretical Science, Princeton University, Princeton, NJ 08544 USA \\ ${ }^{2}$ Joseph Henry Laboratories, Princeton University, Princeton, NJ 08544, USA
}

\begin{abstract}
It has been pointed out by several groups that ekpyrotic and cyclic models generate significant nongaussianity. In this paper, we present a physically intuitive, semi-analytic estimate of the bispectrum. We show that, in all such models, there is an intrinsic contribution to the non-gaussianity parameter $f_{N L}$ that is determined by the geometric mean of the equation of state $w_{e k}$ during the ekpyrotic phase and $w_{c}$ during the phase that curvature perturbations are generated and whose value is $\mathcal{O}(100)$ or more times the intrinsic value predicted by simple slow-roll inflationary models, $f_{N L}^{\text {intrinsic }}=\mathcal{O}(0.1)$. Other contributions to $f_{N L}$, which we also estimate, can increase $\left|f_{N L}\right|$ but are unlikely to decrease it significantly, making non-gaussianity a useful test of these models. Furthermore, we discuss a predicted correlation between the non-gaussianity and scalar spectral index that sharpens the test.
\end{abstract}

\section{INTRODUCTION}

Observations of the cosmic microwave background and large scale structure have provided strong evidence for a nearly scale-invariant spectrum of primordial adiabatic density fluctuations with perhaps a slightly red tilt $[1,2]$. The key cosmological question is: how was this spectrum generated? Two mechanisms are currently known, inflation [3-6] and ekpyrosis [7-11]. Inflation is a period of accelerated expansion following the big bang, characterized by a large Hubble parameter $H$ and an equation of state $w \approx-1$. Ekpyrosis is a period of ultra-slow contraction preceding the big bang, characterized by a small $H$ and $w \gg 1$. One way of distinguishing the two mechanisms is by measuring the spectrum of primordial gravitational waves $[7,8,12]$ whose amplitude, proportional to $H^{2}$, is exponentially different in the inflationary versus ekpyrotic/cyclic models. A second approach, recently emphasized by several groups analyzing different types of ekpyrotic models [13-18], is by measuring the non-gaussian contributions to the density fluctuation spectrum; e.g. the bispectrum, parameterized by the parameter $f_{N L}$ [19], as well as the correlation between $f_{N L}$ and the scalar spectral tilt $n_{s}[18]$.

The purpose of this paper is to provide a physically intuitive semi-analytic estimate of the contributions to the bispectrum that makes clear why measuring both the non-gaussianity and the scalar spectral tilt is a good approach for testing ekpyrotic scenarios of various types, including the cyclic model. We show that ekpyrotic and cyclic models generically produce non-gaussianity of the "local" type [20] with a net value of $f_{N L}$ that is at least an order of magnitude greater than the value predicted by simple slow-roll inflationary models $\left(f_{N L} \lesssim 1\right)$. The prediction is timely because this value is large enough to be detectable in near-future cosmic microwave background studies. The current bound reported by the WMAP collaboration, $-9<f_{N L}<111$ and $0.94<n_{s}<0.99$ at $2 \sigma$ [1] already intrudes on the ekpyrotic range. The uncertainty in $f_{N L}$ will improve modestly with further WMAP data, and then dramatically $\left(\Delta f_{N L} \leq 5\right)$ with the forthcoming Planck satellite mission and large scale structure studies.

The reason why the non-gaussianity in ekpyrotic/cyclic models is generically several orders of magnitude greater than in inflationary models can be traced to the difference in the equation of state during the period that density fluctuations are generated. In standard versions of both models, the density perturbation spectra have their origin in scalar fields $\phi_{i}$ which develop nearly scale invariant perturbations while evolving along an effective potential $V\left(\phi_{i}\right)$. During an inflationary phase, the potential must be nearly constant in order to obtain $w_{\text {inf }} \approx-1$ or, equivalently, $\epsilon_{\text {inf }} \equiv \frac{3}{2}\left(1+w_{\text {inf }}\right) \ll 1$. This means that the inflaton is a nearly free field with nearly gaussian quantum fluctuations. The non-gaussian amplitude depends on the deviation of the potential from perfect flatness or, equivalently, on how close the slow-roll parameter $\epsilon_{\text {inf }}$ and its time variation are to zero. This intuitive argument is consistent with the quantitative expression obtained by Maldacena [21], for example. By contrast, a negative, exponentially steep potential is required to obtain $w_{e k} \gg 1$ or, equivalently, $\epsilon_{e k} \gg 1$ in an ekpyrotic phase, which means that the scalar fields have significant nonlinear self-interactions whose magnitude depends on how large $\epsilon_{e k}$ is. Because the magnitude of $\epsilon_{e k}$ is $\mathcal{O}(100)$ or more times larger than $\epsilon_{i n f}$, the scalar field contribution to the non-gaussianity - which we will call the "intrinsic" part - is significantly larger for ekpyrotic/cyclic models [10, 13-15, 17, 18].

This intuitive argument only refers to the intrinsic contribution to the non-gaussianity, but this is enough to argue why ekpyrotic/cyclic models generically predict $\left|f_{N L}\right|$ to be several orders of magnitude greater than the value in inflationary models. Even if the additional effects discussed below add or subtract from $\left|f_{N L}\right|$ in the ekpyrotic/cyclic model, obtaining a value less than one (that is, in the inflationary range) would only occur through accidental cancellations of independent terms at the two- or three-decimal level, which is highly unnatural [22]. Conversely, it is possible to add features to inflationary models (such as curvatons, non-standard kinetic energy density, etc., with certain parameters) that enhance the non-gaussianity beyond $\left|f_{N L}\right|=1$. However, these are unnecessary embellishments, and, when added, 
can produce virtually arbitrary $f_{N L}$ of either sign.

The predictions can be further refined by taking account of how the scalar field fluctuations are transformed into curvature perturbations. In inflationary models, the scalar field fluctuations directly produce curvature perturbations that are growing modes in an expanding universe. Consequently, the tiny intrinsic non-gaussianity in the scalar fields discussed above translates directly to a tiny non-gaussianity in the curvature fluctuations (if no embellishments are added). In ekpyrotic/cyclic models, the curvature perturbations produced directly by the scalar fields are decaying modes. Hence, the process involves scalar field fluctuations first producing growing mode entropic perturbations during the ekpyrotic phase and, then, converting them to curvature perturbations just before the bounce to an expanding phase [10, 11]. It is notable that the equation of state during this conversion (or $\epsilon_{c}$ ) can be quite different from the equation of state during the ekpyrotic phase (or $\epsilon_{e k}$ ). Since the entire curvature perturbation is produced by this conversion, even the intrinsic contribution is necessarily affected by $\epsilon_{c}$, as well as $\epsilon_{e k}$. In fact, as we will show below, the intrinsic $f_{N L}$ turns out to be proportional to their geometric mean. Hence, the magnitude of $f_{N L}$ in ekpyrotic/cyclic models can be considerably less if the conversion takes places in a kinetic energy dominated phase when $\epsilon_{c}=3 \ll \epsilon_{e k}$, say, rather than in the ekpyrotic phase [18]. This accounts for why the predictions for $f_{N L}$ were found to be significantly greater in the new ekpyrotic model [13-15] compared to the cyclic model [18], although both models predict values much greater than the inflationary case.

The conversion mechanism is also important for determining the sign of the intrinsic contribution to $f_{N L}$. For example, the cyclic model discussed in Ref. [18] produces either sign for the intrinsic $f_{N L}$, whereas the sign is typically negative for the case considered in Ref. [13-15]. Furthermore, the conversion from entropic to curvature perturbations necessarily introduces an additional contribution to the non-gaussianity, which will be analyzed below. We will see that there are substantial regimes where this contribution is smaller in magnitude compared to the intrinsic contribution, but also substantial regimes where it is larger and can even reverse the sign. In the latter case, the net non-gaussianity tends to be so large that it is already ruled out by existing experiments.

This paper is designed to translate the intuitive discussion above into simple semi-analytic estimates, making clear those aspects that are highly model dependent and those that are generic. The conclusions themselves are not so original; for the most part, they appear in earlier papers focusing on particular examples [10, 1315, 17, 18]. Our intent here is more modest: to provide simple expressions that clarify their origin and physical interpretation, so that the significance of forthcoming non-gaussianity tests can be better appreciated. The organization of the paper is as follows: in the next section we set up our notation while reviewing the context of the calculations performed later in the paper. In section III we derive the intrinsic non-gaussianity present in the entropy perturbation in ekpyrotic models. This allows us to present an order-of-magnitude estimate of the resulting curvature perturbation in section IV, before proceeding to discuss various conversion mechanisms in more detail. Section V contains the conclusions that we draw from our analysis.

\section{SETUP}

In the ekpyrotic/cyclic model, the $4 \mathrm{~d}$ effective theory describing the universe around the time of the big bang is given by gravity coupled to two minimally coupled scalar fields with potentials. (This is the case in heterotic Mtheory [23], for example.):

$$
\int \delta^{4} x \sqrt{-g}\left(\frac{1}{2} R-\frac{1}{2} \sum_{i=1}^{2}\left(\partial \phi_{i}\right)^{2}-\sum_{i=1}^{2} V_{i}\left(\phi_{i}\right)\right) .
$$

The scalar field and Friedmann equations are given by

$$
\ddot{\phi}_{i}+3 H \dot{\phi}_{i}+V_{i, \phi_{i}}=0
$$

and

$$
H^{2}=\frac{1}{3}\left[\frac{1}{2} \sum_{i} \dot{\phi}_{i}^{2}+\sum_{i} V_{i}\left(\phi_{i}\right)\right],
$$

where $H=\dot{a} / a, a$ denotes the scale factor, and $V_{i, \phi_{i}}=$ $\left(\partial V_{i} / \partial \phi_{i}\right)$ with no summation implied. In a contracting universe, a growing mode is given by the entropy perturbation, namely the relative fluctuation in the two fields, defined (at linear order) as follows [24]

$$
\delta s \equiv\left(\dot{\phi}_{1} \delta \phi_{2}-\dot{\phi}_{2} \delta \phi_{1}\right) / \dot{\sigma},
$$

where we have defined

$$
\dot{\sigma} \equiv \sqrt{\dot{\phi}_{1}^{2}+\dot{\phi}_{2}^{2}} .
$$

The entropy perturbation is gauge-invariant and it represents the perturbation orthogonal to the background scalar field trajectory, as shown in Fig. 1; see Ref. [25] for its definition to all orders. Its equation of motion, up to second order in field perturbations, is [25]

$$
\begin{aligned}
& \ddot{\delta s}+3 H \dot{\delta} s+\left(V_{s s}+3 \dot{\theta}^{2}\right) \delta s \\
& +\frac{\dot{\theta}}{\dot{\sigma}}\left(\dot{\delta s}{ }^{(1)}\right)^{2}+\frac{2}{\dot{\sigma}}\left(\ddot{\theta}+\dot{\theta} \frac{V_{\sigma}}{\dot{\sigma}}-\frac{3}{2} H \dot{\theta}\right) \delta s^{(1)} \dot{\delta s} s^{(1)} \\
& +\left(\frac{1}{2} V_{s s s}-5 \frac{\dot{\theta}}{\dot{\sigma}} V_{s s}-9 \frac{\dot{\theta}^{3}}{\dot{\sigma}}\right)\left(\delta s^{(1)}\right)^{2}=0,
\end{aligned}
$$

where we have neglected a non-local term that is unimportant in ekpyrotic models [18]. Note that this is a 
closed equation for the entropy perturbation. Here $V_{\sigma}$ denotes a derivative of the potential along the background trajectory and the successive derivatives of the potential with respect to the entropy field are given by

$$
\begin{aligned}
V_{s}= & \frac{1}{\dot{\sigma}}\left(\dot{\phi}_{1} V_{, \phi_{2}}-\dot{\phi}_{2} V_{, \phi_{1}}\right) \\
V_{s s}= & \frac{1}{\dot{\sigma}^{2}}\left(\dot{\phi}_{1}{ }^{2} V_{, \phi_{2} \phi_{2}}-2 \dot{\phi}_{1} \dot{\phi}_{2} V_{\phi_{1} \phi_{2}}+\dot{\phi}_{2}{ }^{2} V_{, \phi_{1} \phi_{1}}\right) \\
V_{s s s}= & \frac{1}{\dot{\sigma}^{3}}\left(\dot{\phi}_{1}{ }^{3} V_{, \phi_{2} \phi_{2} \phi_{2}}-3 \dot{\phi}_{1}{ }^{2} \dot{\phi}_{2} V_{, \phi_{1} \phi_{2} \phi_{2}}\right. \\
& \left.+3 \dot{\phi}_{1} \dot{\phi}_{2}{ }^{2} V_{, \phi_{1} \phi_{1} \phi_{2}}-\dot{\phi}_{2}{ }^{3} V_{, \phi_{1} \phi_{1} \phi_{1}}\right)
\end{aligned}
$$

The angle $\theta$ of the background trajectory is defined by $[24] \cos (\theta)=\dot{\phi}_{1} / \dot{\sigma}, \sin (\theta)=\dot{\phi}_{2} / \dot{\sigma}$ and thus $\dot{\theta}=-V_{s} / \dot{\sigma}$. The parameter $\epsilon$ and the equation of state parameter $w$ are related to the background evolution via

$$
\epsilon \equiv \frac{3}{2}(1+w) \equiv \frac{\dot{\sigma}^{2}}{2 H^{2}},
$$

and the ekpyrotic phase can be characterized by $\epsilon \gg 1$.

\section{INTRINSIC NON-GAUSSIANITY}

The entropy perturbations are generated during the ekpyrotic phase, when the potentials are given by

$$
\sum_{i=1}^{2} V_{i}\left(\phi_{i}\right)=-V_{1} e^{-\int c_{1} \delta \phi_{1}}-V_{2} e^{-\int c_{2} \delta \phi_{2}},
$$

i.e. the potentials are negative and steep. Here we consider $c_{1}=c_{1}\left(\phi_{1}\right)>0$ and $c_{2}=c_{2}\left(\phi_{2}\right)$ to be slowly varying in time, while $V_{1}$ and $V_{2}$ are positive constants. The ekpyrotic phase quickly flattens the universe, so that we can assume a flat Friedmann-Robertson-Walker (FRW) background with line element $\delta s^{2}=-\delta t^{2}+a^{2}(t) \delta \mathbf{x}^{2}$. If the $c_{i}$ are exactly constant and moreover $\left|c_{i}\right| \gg 1$, then the Einstein-scalar equations admit the scaling solution

$$
a=(-t)^{1 / \epsilon}, \quad \phi_{i}=\frac{2}{c_{i}} \ln \left(-\sqrt{c_{i}^{2} V_{i} / 2 t}\right), \quad \frac{1}{\epsilon}=\sum_{i} \frac{2}{c_{i}^{2}} .
$$

This solution describes a very slowly contracting universe with $\epsilon \gg 1$. During the phase in which the entropic perturbations are generated, $\dot{\theta}=0$, which corresponds to a straight background scalar field trajectory. In this case, we define

$$
\dot{\phi}_{2} \equiv \gamma \dot{\phi}_{1}
$$

and, with this notation, $c_{1}=\gamma c_{2}$ and

$$
\epsilon_{e k}=\frac{\left|\gamma c_{1} c_{2}\right|}{2\left(1+\gamma^{2}\right)}
$$

Results are not very sensitive to $\gamma$ so we take $\gamma=\mathcal{O}(1)$ throughout.
It is useful to recast the evolution in terms of the adiabatic and entropic variables $\sigma$ and $s$. Up to unimportant additive constants (that we will fix below), they can be defined by

$$
\begin{aligned}
\sigma & \equiv \frac{\dot{\phi}_{1} \phi_{1}+\dot{\phi}_{2} \phi_{2}}{\dot{\sigma}} \\
s & \equiv \frac{\dot{\phi}_{1} \phi_{2}-\dot{\phi}_{2} \phi_{1}}{\dot{\sigma}}
\end{aligned}
$$

Then we can expand the potential up to third order as follows [26]:

$$
V_{e k}=-V_{0} e^{\sqrt{2 \epsilon} \sigma}\left[1+\epsilon s^{2}+\frac{\kappa_{3}}{3 !} \epsilon^{3 / 2} s^{3}\right],
$$

where $\kappa_{3}$ is of $\mathcal{O}(1)$ for typical potentials (the case of exact exponentials corresponds to $\left.\kappa_{3}=-4 \sqrt{2 / 3}\right)$. The scaling solution (12) can be rewritten as

$$
a(t)=(-t)^{1 / \epsilon} \quad \sigma=-\sqrt{\frac{2}{\epsilon}} \ln \left(-\sqrt{\epsilon V_{0}} t\right) \quad s=0 .
$$

The intrinsic non-gaussianity in the entropy perturbation is produced during the ekpyrotic phase and can be determined from the equation of motion for the entropy field (6), which reduces to

$$
\ddot{\delta s}+3 H \dot{\delta s}+V_{s s} \delta s+\frac{1}{2} V_{s s s}\left(\delta s^{(1)}\right)^{2}=0 .
$$

The last term is

$$
\frac{1}{2} V_{s s s}=-\frac{\kappa_{3}}{2 t^{2}} \sqrt{\epsilon}
$$

and thus the solution, at long wavelengths and up to second order in field perturbations, is given by $[15,18]$

$$
\begin{aligned}
\delta s(t) & =\delta s^{(1)}(t)+\delta s^{(2)}(t) \\
& =\delta s_{\text {end }} \frac{t_{\text {end }}}{t}+\tilde{c}\left(\delta s_{\text {end }} \frac{t_{\text {end }}}{t}\right)^{2},
\end{aligned}
$$

where

$$
\tilde{c}=\frac{\kappa_{3} \sqrt{\epsilon}}{8} .
$$

Thus, the intrinsic non-gaussianity present in ekpyrotic models (second term in (21) above) is of order $\mathcal{O}\left(\sqrt{\epsilon_{e k}}\right)$ and it is due to the steepness of the potentials and the resulting self-interactions of the scalar fields. This is in sharp contrast with inflation, where the intrinsic nongaussianity is extremely small due to the flatness of the potential (or, equivalently, $\epsilon_{\text {inf }} \ll 1$ ).

\section{CONVERSION}

\section{A. An estimate of intrinsic $f_{N L}$}

What is measured is not directly the non-gaussianity present in the entropy perturbation, but the nongaussianity it imprints on the curvature perturbation. 
Thus, it is important to know the strength with which this intrinsic non-gaussianity gets transferred to the curvature perturbation. The time evolution of the curvature perturbation to second order in field perturbations and at long wavelengths is given in FRW time by [25]

$$
\dot{\mathcal{R}}=\frac{2 H}{\dot{\sigma}} \dot{\theta} \delta s+\frac{H}{\dot{\sigma}^{2}}\left[-\left(V_{s s}+4 \dot{\theta}^{2}\right)\left(\delta s^{(1)}\right)^{2}+\frac{V_{, \sigma}}{\dot{\sigma}} \delta s \dot{\delta} s\right] .
$$

where we have omitted a non-local term which can be neglected in ekpyrotic models for the same reason as the non-local term omitted from (6) above, see [18] for details. At linear order, a non-zero entropy perturbation combined with a bending $(\dot{\theta} \neq 0)$ of the background trajectory sources the curvature perturbation on large scales and results in a linear, gaussian curvature perturbation

$$
\begin{aligned}
\mathcal{R}_{L} & =\int_{\Delta t} \frac{2 H}{\dot{\sigma}} \dot{\theta} \delta s^{(1)} \\
& =\int_{\Delta t}-\sqrt{\frac{2}{\epsilon_{c}}} \dot{\theta} \delta s^{(1)},
\end{aligned}
$$

where we denote the duration of the conversion by $\Delta t$ and the sign corresponds to a contracting universe. Thus the strength of conversion is proportional to $1 / \sqrt{\epsilon_{c}}$ and this dependence on the equation of state will have repercussions for the magnitude of the second order correction as well.

Here, as with all the contributions to non-gaussianity discussed in this paper, the fluctuations are generated by the scalar fields with canonical kinetic energy density, so they generate non-gaussianity of the "local" type, as defined in Refs. [19-21]. The local wavelength-independent non-gaussian contribution to $\mathcal{R}$ can then be characterized in terms of the leading linear, gaussian curvature perturbation $\mathcal{R}_{L}$ according to

$$
\mathcal{R}=\mathcal{R}_{L}-\frac{3}{5} f_{N L} \mathcal{R}_{L}^{2}
$$

using the sign convention for wavelength-independent non-gaussianity parameter $f_{N L}$ in [19]. Then the contributions to $f_{N L}$ can be divided into three parts [18]

$$
\begin{aligned}
f_{N L}^{\text {intrinsic }}= & -\frac{5}{3 \mathcal{R}_{L}^{2}} \int_{\Delta t} \frac{2 H}{\dot{\sigma}} \dot{\theta} \delta s^{(2)} \\
f_{N L}^{\text {reflection }}= & \frac{5}{3 \mathcal{R}_{L}^{2}} \int_{r e f} \frac{H}{\dot{\sigma}^{2}}\left[\left(V_{s s}+4 \dot{\theta}^{2}\right)\left(\delta s^{(1)}\right)^{2}\right. \\
& \left.-\frac{V, \sigma}{\dot{\sigma}} \delta s \dot{\delta} s\right] \\
f_{N L}^{\text {integrated }}= & \frac{5}{12 \mathcal{R}_{L}^{2}}\left(\delta s^{(1)}\left(t_{\text {end }}\right)\right)^{2} .
\end{aligned}
$$

$f_{N L}^{\text {intrinsic }}$ arises from the direct translation of the intrinsic non-linearity present in the entropy perturbation into a corresponding non-linearity in the curvature perturbation. By contrast, $f_{N L}^{r e f l e c t i o n}$ and $f_{N L}^{\text {integrated }}$ would be non-zero even if the entropy perturbation were exactly gaussian. Both contributions are due to the non-linear relationship between the curvature perturbation and the entropy perturbation, as expressed in equation (23) - the difference is that $f_{N L}^{\text {integrated }}$ gets generated during the ekpyrotic phase and $f_{N L}^{r e f l e c t i o n}$ during the conversion.

The intrinsic non-gaussianity can be estimated by combining equations (21), (25) and (27):

$$
\begin{aligned}
f_{N L}^{\text {intrinsic }} & \approx \pm \frac{5}{3 \mathcal{R}_{L}^{2}} \int_{\Delta t} \sqrt{\frac{2}{\epsilon_{c}}} \dot{\theta} \tilde{c} \delta s^{(1)^{2}} \\
& \sim \sqrt{\epsilon_{c} \epsilon_{e k}} .
\end{aligned}
$$

It is given by the geometric mean of the $\epsilon$ parameters during the phases of generation and conversion of the perturbations. This is perhaps the most important result because it fixes a rough magnitude for $\left|f_{N L}\right|$ which can only be significantly reduced through accidental cancellations due to the other terms or other effects not included in the present analysis. It explains in a nutshell why the non-gaussianity in ekpyrotic/cyclic models is necessarily more than an order of magnitude greater than in simple inflation models, and it explains why the equation of state during conversion can have a significant quantitative effect on the prediction.

To go beyond this qualitative estimate to a more precise one, we need to take account of the details of the conversion mechanism. We will now discuss the various possibilities considered in the literature, namely conversion during the ekpyrotic phase, after the ekpyrotic phase during kinetic energy domination, and conversion after the big bang by modulated preheating.

\section{B. Conversion during kinetic energy domination}

In the original ekpyrotic and cyclic models, the phase dominated by the steep, ekpyrotic potential $V(\phi)$ comes to an end (at $t=t_{\text {end }}<0$ ) before the big crunch/big bang transition $(t=0)$, and the universe becomes dominated by the kinetic energy of the scalar fields. Consequently, the equation of state at $t=t_{\text {end }}$ changes from $\epsilon_{e k} \gg 1$ to $\epsilon=3$ (corresponding to $w \rightarrow 1$, the equation of state for a kinetic energy dominated universe). In this subsection, we consider the case where the conversion from entropic to curvature perturbations occurs during this kinetic energy dominated phase. This occurs naturally in the heterotic M-theory embedding of the cyclic model because the negative-tension brane bounces off a spacetime singularity [27] - creating a bend in the trajectory in field space in the $4 \mathrm{~d}$ effective theory - before it collides with the positive-tension brane (the big crunchbig bang transition). This bending of the trajectory automatically induces the conversion of entropy to curvature perturbations [11]. Here we will not restrict the analysis to this particular example, but we will consider the general situation in which the scalar field trajectory bends in a smooth way during the phase of kinetic energy domination following an ekpyrotic phase. Before proceeding, 


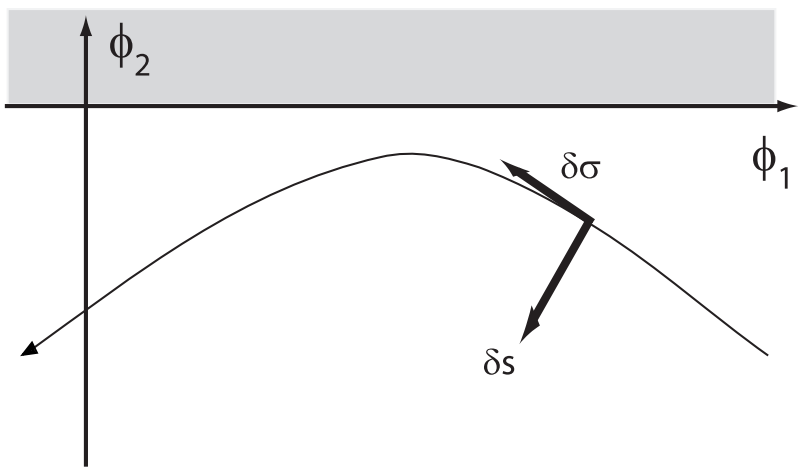

FIG. 1: The trajectory in field space reflects off a boundary at $\phi_{2}=0$. The entropy perturbation, denoted $\delta s$, is orthogonal to the trajectory. The bending causes the conversion of entropy modes into adiabatic modes $\delta \sigma$, which are perturbations tangential to the trajectory.

we should remark that due to the instability of the scaling solution during the ekpyrotic phase $[11,28]$, we must assume the background trajectory is localized very close to the ridge of the two-field ekpyrotic potential at the beginning of the ekpyrotic phase in order for the conversion not to happen already during the ekpyrotic phase. How this initial condition can arise in a cyclic model is beyond the scope of the present paper.

We can immediately perform an order-of-magnitude estimate of the non-gaussianity since during the kinetic phase $\epsilon_{c}=3$, and so (30) would lead us to expect $f_{N L}$ to be of order

$$
f_{N L} \sim \sqrt{\epsilon_{e k}} \sim \mathcal{O}\left(c_{1}\right)
$$

We will treat the bend as if only one field reflects $\left(\phi_{2}\right)$, and we will consider the case where $\dot{\theta}>0$, see Fig. 1 . Other cases can be related to these representative examples by changing the coordinate system in field space appropriately. In the heterotic M-theory example, the reflection occurs because $\phi_{2}$ comes close to a boundary of moduli space $\left(\phi_{2}=0\right)$ and is forced to bounce [27]. For the purposes of this study, we will treat the reflection as being due to a potential, $V^{R}\left(\phi_{2}\right)$, an additional contribution unrelated to the exponential potentials that were dominant during the ekpyrotic phase (but which are negligible during the kinetic energy dominated phase).

It is important to know the evolution of the entropy perturbation during the process of conversion. If there is a phase of pure kinetic energy domination before the conversion, then the background scalar field trajectory is also a straight line during this phase, but with the potentials being irrelevant. The equation of motion reduces to

$$
\ddot{\delta s}+\frac{1}{t} \dot{\delta s}=0
$$

and by matching onto the ekpyrotic solution and its first time derivative at $t=t_{\text {end }}$ we find

$$
\begin{aligned}
\delta s(t) & =\delta s^{(1)}(t)+\delta s^{(2)}(t) \\
& =\delta s_{\text {end }}\left(1+\ln \frac{t_{\text {end }}}{t}\right)+\tilde{c} \delta s_{\text {end }}^{2}\left(1+2 \ln \frac{t_{\text {end }}}{t}\right)(33)
\end{aligned}
$$

Incidentally, note that

$$
t \frac{\dot{\delta s}}{\delta s} \sim \frac{1}{\ln (-t)}
$$

during the kinetic phase, while during the ekpyrotic phase $t \dot{\delta} s \sim \delta s$. This observation will simplify our analysis later on.

During the conversion, even though the kinetic energy of the scalar fields is still the dominant contribution to the total energy, the potential $V^{R}\left(\phi_{2}\right)$ that causes the bending has a significant influence on the evolution of the entropy perturbation. To analyze this, we will approximate (as in [16]) the bending of the trajectory to be gradual by taking $\dot{\theta}$ constant and non-zero for a period of time $\Delta t$, starting from $t=t_{r e f}$. Note that, assuming the total angle of bending is $\mathcal{O}(1)$ radian, we have $|\dot{\theta}| \approx 1 /\left|t_{\text {ref }}\right|$ in this case. Then one can relate the derivatives of the potential to expressions involving $\dot{\theta}$, for example

$$
V_{s s}^{R}=-2 \dot{\theta}^{2}+\frac{\dot{\theta}}{\gamma t} .
$$

Assuming a gradual conversion $\left(\Delta t \sim t_{r e f}\right)$, we can ignore higher derivatives of $\theta$. (In [18] it was shown that sharp transitions lead to unacceptably large values of $f_{N L}$; hence these cases are of less phenomenological interest.) To satisfy the constraint on the amplitude of the curvature perturbation obtained by the WMAP observations, we set $t_{r e f} \approx-10^{3} M_{P l}^{-1}[11]$. At linear order, the equation of motion (6) then reads

$$
\ddot{\delta s}^{(1)}+3 H \dot{\delta s}^{(1)}+\left(\dot{\theta}^{2}+\frac{1}{\gamma t} \dot{\theta}\right) \delta s^{(1)}=0,
$$

As indicated by equation (34), we can set $\dot{\delta s}^{(1)}=0$ as a first approximation and, thus, neglect the damping term in the equation of motion. Also, we will simply evaluate the coefficient of the last term midway through the reflection, and define

$$
\omega \equiv \dot{\theta} \sqrt{1+\frac{1}{\dot{\theta} \gamma\left(t_{r e f}+\Delta t / 2\right)}} .
$$

For a gradual reflection $\omega \approx(2-3) \dot{\theta}$. Then the solution for the linear entropy perturbation is

$$
\delta s^{(1)}=\delta s^{(1)}\left(t_{r e f}\right) \cos \omega\left(t-t_{r e f}\right) .
$$

Instead of continuing to grow logarithmically, the entropy perturbation actually falls off during the conversion, see Fig. 2. This has the consequence that the conversion from entropy to curvature perturbations is less efficient 


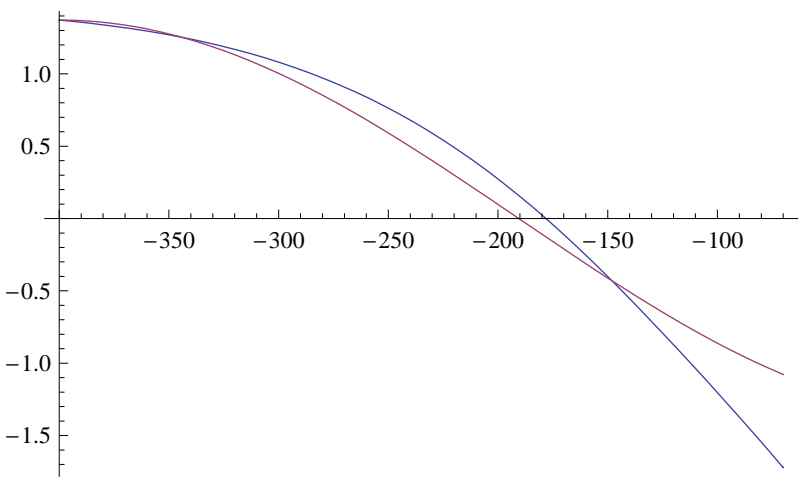

FIG. 2: The evolution of the linear entropy perturbation during conversion: the solid (blue) line shows the actual evolution calculated numerically, while the dashed (purple) line shows the approximate solution (38) with $\omega=3, \dot{\theta}=-1 / t_{\text {ref }}$ and $t_{r e f}=-400 M_{P l}^{-1}$. For the purposes of illustration, $\delta s^{(1)}\left(t_{r e f}\right)$ has been normalized to 1 .

than one might have naively thought. From (25), we can estimate

$$
\mathcal{R}_{L}=-\sqrt{\frac{2}{3}} \dot{\theta} \int_{\Delta t} \delta s^{(1)}=-\sqrt{\frac{2}{3}} \frac{\dot{\theta}}{\omega} \delta s^{(1)}\left(t_{r e f}\right) \sin \omega \Delta t,
$$

where we have used $\epsilon_{c} \approx 3$, which is a good approximation for subdominant reflections (and acceptable for the estimating purposes for dominant ones). Since $\omega \Delta t \approx 3$, the entropy perturbation evolves over nearly a half-cycle and consequently $\sin \omega \Delta t$ is a small factor which we will take to be about $1 / 3$ in our estimates, which fits well with numerical results.

We also need to know the evolution of the second order entropy perturbation during the time of conversion. Using the same approximations as above (implying that we can neglect $V_{s s s}$ compared to $\dot{\theta} V_{s s} / \dot{\sigma}$ and $\dot{\theta}^{3} / \dot{\sigma}$ by the time the conversion is underway), the equation of motion (6) simplifies to

$$
\ddot{\delta s}^{(2)}+\omega^{2} \delta s^{(2)}-\frac{\dot{\theta}}{\dot{\sigma}} \omega^{2}\left(\delta s^{(1)}\right)^{2}=0,
$$

where $\delta s^{(1)}$ is given in (38). Putting $\dot{\theta} \approx \dot{\sigma}$ at the start of the reflection and keeping in mind that we impose the boundary condition $\dot{\delta s}^{(2)} \approx 0$, the solution for the second order entropy perturbation is given by

$$
\begin{aligned}
\delta s^{(2)}= & \delta s^{(2)}\left(t_{r e f}\right) \cos \left[\omega\left(t-t_{r e f}\right)\right] \\
& +\frac{1}{12}\left(\delta s^{(1)}\left(t_{r e f}\right)\right)^{2}\left(-4 \cos \left[\omega\left(t-t_{r e f}\right)\right]\right. \\
& +4 \cos ^{4}\left[\omega\left(t-t_{r e f}\right)\right]+9 \sin ^{2}\left[\omega\left(t-t_{r e f}\right)\right] \\
& \left.+\sin \left[\omega\left(t-t_{r e f}\right)\right] \sin \left[3 \omega\left(t-t_{r e f}\right)\right]\right) .
\end{aligned}
$$

At large $\epsilon_{e k}$ (or, equivalently, large $\left.|\tilde{c}|\right)$, the second order entropy perturbation falls off in the same way as the linear perturbation, but at small $|\tilde{c}|$ there are significant corrections to this behavior. We will also need the integral

$$
\begin{aligned}
C^{-2} \int_{\Delta t} \delta s^{(2)}= & \frac{\left(1+2 \ln \left(t_{\text {end }} / t_{\text {ref }}\right)\right)}{\left(1+\ln \left(t_{\text {end }} / t_{\text {ref }}\right)\right)^{2}} \frac{\sin (\omega \Delta t)}{\omega} \tilde{c} \\
& +\frac{\Delta t}{2}-\frac{\sin (\omega \Delta t)}{3 \omega}-\frac{\sin (2 \omega \Delta t)}{12 \omega}
\end{aligned}
$$

where $C=\delta s^{(1)}\left(t_{r e f}\right)$. In all cases of interest, the last two terms are negligible.

We are finally in a position to evaluate the various contributions to the non-linearity parameter $f_{N L}$. The intrinsic contribution, defined in (27), becomes

$$
f_{N L}^{\text {intrinsic }} \approx A \kappa_{3} \sqrt{\epsilon_{e k}}+B,
$$

with

$$
\begin{aligned}
A & =\frac{5 \omega\left(1+2 \ln \left(t_{\text {end }} / t_{\text {ref }}\right)\right)}{8 \sqrt{6} \dot{\theta}\left(1+\ln \left(t_{\text {end }} / t_{\text {ref }}\right)\right)^{2} \sin (\omega \Delta t)} \\
B & =\frac{5 \omega^{2}}{2 \sqrt{6} \dot{\theta}^{2} \sin ^{2}(\omega \Delta t)} .
\end{aligned}
$$

Eq. (43) is one of our key results because it shows that the essential contribution scales in a simple way with $\epsilon_{e k}$ and has a value that exceeds the total $f_{N L}$ for simple inflationary models by more than an order of magnitude. As suggested by Eq. (30), the first term in $f_{N L}^{\text {intrinsic }}$ can be re-expressed as $\mathcal{O}\left(\sqrt{\epsilon_{c} \epsilon_{e k}}\right)$ with $\epsilon_{c}=3$. This contribution comes directly from the non-zero $\delta s^{(2)}$ generated during the ekpyrotic phase, which is due to the third derivative w.r.t. $s$ of the ekpyrotic potential. It is interesting to note that, in the case where $V_{s s s}=0$ or $\kappa_{3}=0, f_{N L}^{\text {intrinsic }}$ is nevertheless non-negligible because of the positive offset $B$ generated by the linear entropy perturbation $\delta s^{(1)}$ during conversion, the piece proportional to $\left(\delta s^{(1)}\right)^{2}$ in (41).

Using the definition (28) together with (35) it is straightforward to see that for conversion during the kinetic phase, $f_{N L}^{r e f l e c t i o n}$ is always negative, and it can be estimated as

$$
\begin{aligned}
f_{N L}^{\text {reflection }} & \approx \frac{5}{6 \mathcal{R}_{L}^{2}} \int_{\Delta t}\left(2 \dot{\theta}^{2} t+\frac{\dot{\theta}}{\gamma}\right)\left(\delta s^{(1)}\right)^{2} \\
& \approx-\frac{15 \omega^{2}}{8 \dot{\theta}^{2} \sin ^{2}(\omega \Delta t)} \frac{\left|t_{r e f}+\Delta t / 2\right|}{\Delta t},
\end{aligned}
$$

where we have used $\gamma t_{r e f} \dot{\theta} \approx 1$ and

$$
\int_{\Delta t} t \sin ^{2} \omega\left(t-t_{r e f}\right) \approx \frac{1}{2}\left(t_{r e f}+\frac{\Delta t}{2}\right) \Delta t,
$$

which is a valid approximation since $\delta s^{(1)}$ evolves over approximately a half-cycle. We have neglected the contribution due to the term proportional to $\delta s \dot{\delta} s$, since it is suppressed on account of (34). The integrated contribution to $f_{N L}$ generated during the ekpyrotic phase gives 
an additional contribution of

$$
\begin{aligned}
f_{N L}^{\text {integrated }} & =\frac{5}{12 \mathcal{R}_{L}^{2}}\left(\delta s^{(1)}\left(t_{\text {end }}\right)\right)^{2} \\
& \approx \frac{5 \omega^{2}}{8 \dot{\theta}^{2}\left(1+\ln \left(t_{\text {end }} / t_{\text {ref }}\right)\right)^{2} \sin ^{2}(\omega \Delta t)}
\end{aligned}
$$

Note that neither the reflected nor the integrated contributions depend on $\epsilon_{e k}$; they both simply shift the final result by a number depending on the sharpness of the transition and the duration of the purely kinetic phase respectively.

The total $f_{N L}$ is the sum of all the above contributions. Since we are only considering gradual conversions, we expect the ratio $\left|t_{r e f}+\Delta t / 2\right| / \Delta t$ to lie between 1 and 2 . Also, the duration of the pure kinetic phase between the end of the ekpyrotic phase and the conversion is necessarily rather short, so that we expect $\ln \left(t_{\text {end }} / t_{\text {ref }}\right) \lesssim \mathcal{O}(1)$.

Putting everything together, we get the following estimates:

$$
\begin{aligned}
f_{N L}^{\text {intrinsic }} & \approx 10 \tilde{c}+50 \\
f_{N L}^{\text {reflection }} & \approx-50 \\
f_{N L}^{\text {integrated }} & \approx 5 .
\end{aligned}
$$

The analytic estimate for $f_{N L}^{r e f l e c t i o n}$ suggests a range that could extend to -100 , but the value above is more consistent with the exact numerical calculations for a wide range of parameters. Altogether, we end up with the following fitting formula

$$
\begin{aligned}
f_{N L}^{t o t a l} & \approx 10 \tilde{c}+5 \\
& \approx \frac{3}{2} \kappa_{3} \sqrt{\epsilon_{e k}}+5 .
\end{aligned}
$$

Fig. 3 shows that this formula agrees well with the results from exact numerical calculations. In particular, we have been able to estimate the slope, and hence the dependence on $\kappa_{3} \sqrt{\epsilon_{e k}}$, rather accurately.

\section{Conversion during the ekpyrotic phase}

In the "new ekpyrotic" scenario $[13,14,29]$, the conversion of entropy to curvature perturbations takes place because the background trajectory switches from the twofield unstable scaling solution to a single-field attractor solution; in other words, the trajectory starts out close to the ridge of the two-field potential and then falls off one of the steep sides, either because of the initial conditions or due to an additional feature in the potential. Adding a feature to the potential makes little difference to the results as long as the reflection remains gradual, so in fact we will not consider an additional potential $V^{R}$ in this section. Since the conversion happens during the ekpyrotic phase, i.e. while the ekpyrotic potentials are relevant, $\epsilon_{c}=\epsilon_{e k}$, and from (30) we expect the nongaussianity to be of order

$$
f_{N L} \sim \epsilon_{e k} \sim \mathcal{O}\left(c_{1}^{2}\right) .
$$

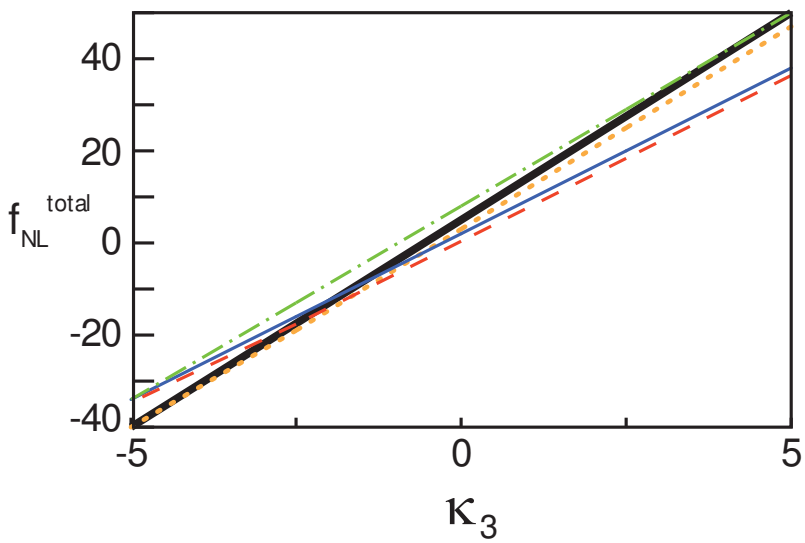

FIG. 3: A comparison of the results from numerical calculations with the fitting formula given in Eq. (52) and indicated by the thick black line. Here we have fixed the value of $\epsilon_{e k}=36$. The plot confirms that $f_{N L}$ then grows linearly with $\kappa_{3}$, in good agreement with (52). The sample models (dashed and dotted lines) are representative of the range of models and parameters shown in [18]. We have similarly checked the dependence on $\sqrt{\epsilon}$ when the value of $\kappa_{3}$ is kept fixed.

As shown by Koyama et al.[15], the $\delta N$ formalism is well suited to treating this case, with the result that the total local non-linearity parameter is given by

$$
f_{N L}=-\frac{5}{12} c_{j}^{2}
$$

where the index $j$ corresponds to the field that becomes frozen in the late-time single-field solution (here we have reverted to the potential form (11), as it is better suited for studying conversion during the ekpyrotic phase). As a check, we have performed the direct integration of the equations of motion numerically [30], and we find results that are in good agreement with the above analytic estimate. Note that the sign of $f_{N L}$ is always negative for these cases.

A qualitative understanding of this result can be achieved in the present context as follows: from (27) we can see that, since $H / \dot{\sigma}$ is negative and approximately constant, the sign of $f_{N L}^{\text {intrinsic }}$ is given by the sign of $\int \dot{\theta} \delta s^{(2)}$. In practice, numerical simulations indicate that, for conversion during the ekpyrotic phase, we have

$$
|\dot{\theta}| \approx \frac{1}{10|t|}
$$

during most of the conversion, but with $\dot{\theta}$ growing faster towards the end. We will use this numerical input to guide our analysis. The sign of $f_{N L}^{\text {intrinsic }}$ is essentially determined by the sign of $\dot{\theta} \delta s^{(2)}$ towards the end of the period of conversion. Naively it is difficult to perform a purely analytic estimate of $f_{N L}$ in the current scheme, since all the terms in the equation of motion (6) go as $c_{1} t^{-4}$; however, equation (55) tells us that

$$
\left|V_{s s}\right|=2 / t^{2} \gg \dot{\theta}^{2},
$$




\begin{tabular}{|c|c||c||c|}
\hline$c_{1}$ & $c_{2}$ & $f_{N L, \delta N}$ & $f_{N L}$ \\
\hline 10 & 10 & -41.67 & -39.95 \\
10 & 15 & -41.67 & -40.45 \\
10 & 20 & -41.67 & -40.62 \\
15 & 10 & -93.75 & -91.01 \\
15 & 15 & -93.75 & -92.11 \\
15 & 20 & -93.75 & -92.49 \\
20 & 10 & -166.7 & -162.5 \\
20 & 15 & -166.7 & -164.4 \\
20 & 20 & -166.7 & -165.1 \\
\hline
\end{tabular}

TABLE I: Ekpyrotic conversion: the values of $f_{N L}$ estimated by the $\delta N$ formalism compared to the numerical results obtained by directly integrating the equations of motion.

and so there are surprisingly few terms in the equation of motion for $\delta s^{(2)}$ that are actually important during (most of) the time of conversion. In fact, to a first approximation, we are simply left with

$$
\ddot{\delta s^{(2)}}=\left(-\frac{1}{2} V_{s s s}+\frac{\dot{\theta}}{\dot{\sigma}} V_{s s}\right)\left(\delta s^{(1)}\right)^{2} .
$$

$V_{\text {sss }}$ decreases in importance as the single-field scaling solution is reached. Thus, even though the $V_{\text {sss }}$ term determines the initial evolution of $\delta s^{(2)}$, eventually the $V_{s s}$ term dominates. Then, since $V_{s s}<0$ it is easy to see that the sign of $\delta s^{(2)}$ is always driven to be opposite to that of $\dot{\theta}$, and consequently $f_{N L}^{\text {intrinsic }}$ is negative in all cases.

Since $f_{N L}^{\text {reflection }}$ is proportional to $\mathcal{R}_{L}^{-2}$, it provides a contribution of the same order $\mathcal{O}\left(\epsilon_{e k}\right)$. Using (56), it is straightforward to see that the part of $f_{N L}^{r e f l e c t i o n}$ that is proportional to $(\delta s)^{2}$ is always positive, while the part proportional to $\delta s \dot{\delta s}$ is always negative. This implies that there will be a competition between the two contributions. Numerical integration then shows that the part proportional to $(\delta s)^{2}$ approximately cancels out $f_{N L}^{i n t r i n s i c}$, while the $\delta s \dot{\delta s}$ part by itself is very close in numerical value to the final answer. In all cases $f_{N L}^{\text {integrated }}$ is completely negligible.

Consequently, the total $f_{N L}$ turns out to be negative and moreover in good agreement with the $\delta N$ result (54) [15], as shown in Table I. Clearly, the $\delta N$ formalism provides a fast and elegant derivation of non-gaussianity for conversion during the ekpyrotic phase that agrees well with direct integration of the equations of motion. (Note, however, no analogous $\delta N$ approach applies to conversion in the kinetic energy dominated phase.)

The conclusion that $f_{N L}$ is substantially less than zero differs from Ref. [16]; their approximation method was incomplete though, since the term proportional to $\delta s \dot{\delta s}$ in (23) was not included. As shown above, this term typically contributes significantly to the final result. Also, the evolution of the entropy perturbation during conversion was neglected, which meant, for example, that values of $f_{N L}^{\text {intrinsic }}$ of either sign were obtained. Our results here show that their approximations were too crude, and that $f_{N L}$ is generally negative with $f_{N L} \lesssim-20$. This is inconsistent with current limits by roughly $3 \sigma$ [1].

Hence, we conclude that models with conversion during the ekpyrotic phase $\left(\epsilon_{c}=\epsilon_{e k} \gg 1\right)$ are difficult to accommodate with current observations in contrast to models in which conversion occurs during the kinetic energy dominated phase $\left(\epsilon_{c}=3\right)$.

\section{Conversion after the crunch/bang transition}

It has recently been proposed by Battefeld [17] that, instead of converting entropy perturbations into curvature perturbations before the big crunch/big bang transition, the conversion could occur during the phase shortly following the bang through modulated reheating. The concept is that massive matter fields are produced copiously at the brane collision and dominate the energy density immediately after the bang. The massive fields are assumed to couple to ordinary matter with a strength proportional to $h(\delta s)$, so that their decay into ordinary matter occurs at slightly different times depending on the value of $\delta s$. In this way, the ordinary matter perturbations inherit the entropic perturbation spectrum. Since the conversion happens while $\epsilon_{c} \approx 3$, we can expect an intrinsic contribution to $f_{N L}$ of

$$
f_{N L} \sim \sqrt{\epsilon_{e k}} \sim \mathcal{O}\left(c_{1}\right)
$$

i.e. $f_{N L}$ is of the same order as in the case of conversion during the kinetic phase preceding the big crunch. Moreover, as shown in [17], no significant additional contributions to $f_{N L}$ are expected. Therefore, models of this type are subject to roughly the same observational constraints as the models where the conversion happens during the kinetic phase before the bang and where the intrinsic contribution to the non-gaussianity is dominant.

Note however that a detailed prediction is made difficult by the fact that $h$ is an unknown function of $\delta s$. Indeed, the entropy perturbations are converted with an efficiency [17]

$$
e=\frac{3}{2} \frac{\left|h_{, s}\right|}{h} .
$$

Since the non-linearity in the entropy field is of magnitude $\tilde{c}$, this implies a non-linearity in the curvature perturbation given by

$$
f_{N L} \approx \pm \frac{\tilde{c}}{e},
$$

where the \pm sign reflects our ignorance of the sign of $h_{, s}$ and of the direction of bending of the scalar field trajectory. Thus, in the absence of a more detailed model, we cannot go beyond this rough order-of-magnitude estimate at present. 


\section{CONCLUSIONS}

Our analysis can be summarized in a few rules of thumb:

- The intrinsic contribution to $\left|f_{N L}\right|$ is proportional to the geometric mean of $\epsilon_{e k}$ and $\epsilon_{c}$, which is at least two orders of magnitude greater than in simple inflationary models (where $\left|f_{N L}^{\text {intrinsic }}\right|=$ $\mathcal{O}(0.1))$. When all contributions are considered, the total $f_{N L}$ is generically more than an order of magnitude greater than in simple inflationary models, $\left(\right.$ where $\left.f_{N L}^{\text {total }}=\mathcal{O}(1)\right)$.

- For a fixed value of $\kappa_{3}$, the value of $\left|f_{N L}^{\text {total }}\right|$ is correlated with the spectral tilt: smaller $\left|f_{N L}^{\text {total }}\right|$ implies smaller $\epsilon_{e k}$, which tends to make the spectral tilt bluer. Current limits on $f_{N L}$ fit well with limits on the spectral tilt for the simplest models with conversion during the kinetic energy driven phase before the bang or during reheating after the big bang.

- Models in which the conversion occurs during a phase with larger $\epsilon_{c}$ produce a larger intrinsic $\left|f_{N L}\right|$ and are more difficult to fit with the current observed limits on $f_{N L}$ and spectral tilt. In particular, models with conversion in the ekpyrotic phase favor $\left|f_{N L}\right|$ large and negative.

- Cases in which the intrinsic contribution to $f_{N L}$ is much smaller than the reflection plus integrated contributions produces very large values of $\left|f_{N L}\right| \gg$ 100 that are inconsistent with current observational bounds. This includes all cases where the conversion is sharp.

The analysis suggests a useful characteristic plot for differentiating cosmological models: $f_{N L}$ versus tilt. Figure 4 illustrates the prediction for a cyclic model in which the conversion from entropic to curvature perturbations occurs in the kinetic energy dominated epoch following the ekpyrotic phase. Here we keep the skewness $\kappa_{3}$ of the potential fixed, as we vary its steepness $\epsilon$. The prediction is a swath whose width is largely due to the uncertainty in the trajectory, parameterized by $\gamma$. Although the swath includes $f_{N L}$ near zero, positive $f_{N L}$ between 10 and 100 is preferred for tilts in the range suggested by WMAP 5 . The prediction for simple inflationary models is confined to the narrow band $\Delta f_{N L} \lesssim 1$ around zero.

The results are surprisingly predictive. If observations of $f_{N L}$ lie in the range predicted by the intrinsic contribution of either inflationary or ekpyrotic/cyclic models, it is reasonable to apply Occam's razor and Bayesian analysis to favor one cosmological model over the other. Combining with measurements of the spectral tilt significantly sharpens the test. The current observational bounds obtained by the WMAP satellite are still inconclusive, but it is clear from the estimates presented here that nongaussianity should be detected by the Planck satellite if

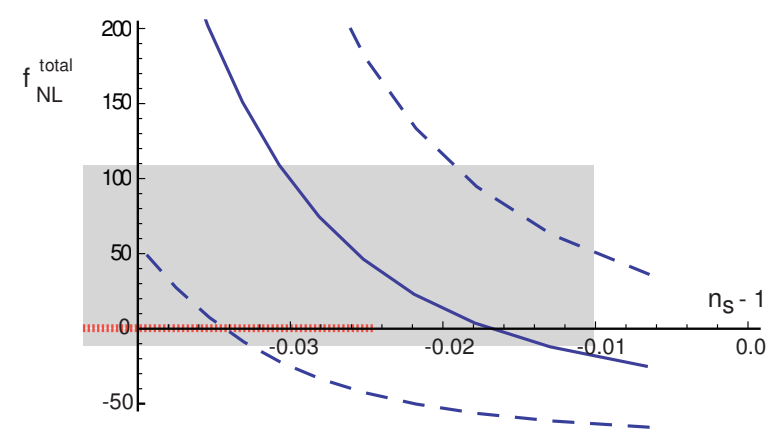

FIG. 4: A plot for characterizing the correlation between $\left|f_{N L}\right|$ and scalar spectral tilt, $n_{s}-1$, here illustrated for the case of the cyclic model in which the conversion from entropic to curvature perturbations occurs during the kinetic energy dominated phase just before the big crunch/big bang transition. Different curves correspond to different fixed amounts of skewness $\kappa_{3}$ in the potential (the central curve corresponds to $\kappa_{3}=4 \sqrt{2 / 3}$ ), while we vary the steepness of the potential $\epsilon_{e k}$. The curves show the general trend that $\left|f_{N L}\right|$ increases as the spectrum becomes redder. Simple inflationary models correspond to the narrow horizontal hashed (red) strip with $\left|f_{N L}\right| \lesssim 1$. The shaded rectangle represents the current observational constraints on $f_{N L}$ and tilt (95\% confidence) from WMAP5 [1].

the ekpyrotic/cyclic model is correct. At the same time, this provides a strong incentive to further refine other methods of measuring non-gaussianity, such as looking for evidence in measurements of the large scale structure of the universe.

\section{Acknowledgements}

We would like to thank Thorsten Battefeld, Evgeny Buchbinder, Justin Khoury, Burt Ovrut, Neil Turok and David Wands for helpful and stimulating discussions, and in particular Kazuya Koyama for discussions and detailed comparisons with the $\delta N$ formalism and Sébastien Renaux-Petel for pointing out a sign error in $\tilde{c}$ in an earlier version of this paper. We would also like to thank the Perimeter Institute for hospitality while this work was completed. This work is supported in part by the US Department of Energy grant DE-FG02-91ER40671.

[1] E. Komatsu et al. [WMAP Collaboration], arXiv:0803.0547 [astro-ph]. 
(2006) [arXiv:astro-ph/0604335].

[3] A. H. Guth and S. Y. Pi, Phys. Rev. Lett. 49, 1110 (1982).

[4] S. W. Hawking, Phys. Lett. B 115, 295 (1982).

[5] A. A. Starobinsky, Phys. Lett. B 117, 175 (1982).

[6] J. M. Bardeen, P. J. Steinhardt and M. S. Turner, Phys. Rev. D 28, 679 (1983).

[7] J. Khoury, B. A. Ovrut, P. J. Steinhardt and N. Turok, Phys. Rev. D 64, 123522 (2001) [arXiv:hep-th/0103239].

[8] P. J. Steinhardt and N. Turok, Science 296, 1436 (2002); Phys. Rev. D65, 126003 (2002).

[9] A. J. Tolley, N. Turok and P. J. Steinhardt, Phys. Rev. D 69, 106005 (2004); P. L. McFadden, N. Turok and P. J. Steinhardt, Phys. Rev. D 76, 104038 (2007).

[10] A. Notari and A. Riotto, Nucl. Phys. B 644, 371 (2002); F. Di Marco, F. Finelli and R. Brandenberger, Phys. Rev. D 67, 063512 (2003).

[11] J. L. Lehners, P. McFadden, N. Turok and P. J. Steinhardt, Phys. Rev. D 76, 103501 (2007) [arXiv:hepth/0702153].

[12] L. A. Boyle, P. J. Steinhardt and N. Turok, Phys. Rev. D 69, 127302 (2004) [arXiv:hep-th/0307170].

[13] E. I. Buchbinder, J. Khoury and B. A. Ovrut, Phys. Rev. D 76, 123503 (2007).

[14] P. Creminelli and L. Senatore, JCAP 0711, 010 (2007).

[15] K. Koyama, S. Mizuno, F. Vernizzi and D. Wands, JCAP 0711, 024 (2007).

[16] E. I. Buchbinder, J. Khoury, and B. A. Ovrut (2007), arXiv:0710.5172 [hep-th].

[17] T. Battefeld, Phys. Rev. D 77, 063503 (2008) [arXiv:0710.2540 [hep-th]].
[18] J. L. Lehners and P. J. Steinhardt, Phys. Rev. D 77, 063533 (2008) [arXiv:0712.3779 [hep-th]].

[19] E. Komatsu and D. N. Spergel, arXiv:astro-ph/0012197; Phys. Rev. D 63, 063002 (2001).

[20] D. Babich, P. Creminelli and M. Zaldarriaga, JCAP 0408, 009 (2004) [arXiv:astro-ph/0405356].

[21] J. M. Maldacena, JHEP 0305, 013 (2003).

[22] Our conclusions about ekpyrotic models may not apply to the method for generating curvature perturbations in ekpyrotic models suggested in N. Turok, B. Craps and T. Hertog, arXiv:0711.1824 [hep-th]; B. Craps, T. Hertog and N. Turok, arXiv:0712.4180 [hep-th].

[23] J. L. Lehners, P. McFadden and N. Turok, Phys. Rev. D 76, 023501 (2007) [arXiv:hep-th/0612026].

[24] C. Gordon, D. Wands, B. A. Bassett, and R. Maartens, Phys. Rev. D63, 023506 (2001).

[25] D. Langlois and F. Vernizzi, JCAP 0702, 017 (2007).

[26] E. I. Buchbinder, J. Khoury and B. A. Ovrut, JHEP 0711, 076 (2007) [arXiv:0706.3903 [hep-th]].

[27] J. L. Lehners, P. McFadden and N. Turok, Phys. Rev. D 75, 103510 (2007) [arXiv:hep-th/0611259]; J. L. Lehners and N. Turok, Phys. Rev. D 77, 023516 (2008) [arXiv:0708.0743 [hep-th]].

[28] A. J. Tolley and D. H. Wesley, JCAP 0705, 006 (2007) [arXiv:hep-th/0703101].

[29] K. Koyama and D. Wands, JCAP 0704, 008 (2007); K. Koyama, S. Mizuno and D. Wands, Class. Quant. Grav. 24, 3919 (2007).

[30] In their paper, Koyama et al. also performed a numerical check within the framework of the $\delta N$ formalism. 